\title{
Radial Force Control for Triple Three-Phase Sectored SPM Machines. Part I: Machine Model
}

\author{
G. Sala, D. Gerada, C. Gerada, IEEE Member, A. Tani
}

\begin{abstract}
The radial force control technique for a triple three-phase Surface Permanent Magnet (SPM) machine is investigated in this paper. The machine has a nine-phase winding arranged in three sectors and supplied by three different Voltage Source Inverters (VSI). A machine model is developed, based on the multi space vector approach. The multi space vector current control technique is exploited to control the torque and the radial force. The radial force control can be useful to compensate for a bearing fault or for a rotor eccentricity. Finite element simulations are used to validate the model and the control technique. Finally, criticalities of the control and modelling aspects are discussed.
\end{abstract}

Index Terms - Analytical models, brushless machines, fault tolerance, fault tolerant systems, force, force control, machine vector control, permanent magnet machines.

\section{INTRODUCTION}

$\mathrm{M}$ ULTIPHASE machines have a series of benefits, when compared to conventional three-phase ones, in terms of performance and fault tolerant behaviour. Owing to the higher number of phases, the degrees of freedom increase allows controlling more harmonics of the air-gap field. The multiphase current control can be exploited to reduce the torque ripple related to the higher harmonic fields, to levitate the rotor in a bearingless configuration, to define a sensorless machine control, to detect machine faults and implement fault tolerant controls, and for other purposes related to the various machine topologies [1]-[3].

The radial force can be controlled with two motor topologies according to their winding configuration: dual set and single set winding. While in the first category the radial force is produced by the design of a specific winding with a different pole pair number, in the latter one it is generated by the multiphase machine control. Indeed, in multiphase machines it is possible to inject current sequences in order to control both even and odd field harmonics, and so both the radial force and the torque [4].

In critical systems or when high reliability is required, as in More Electric Aircraft (MEA) applications, machine fault tolerant controls are needed. The possibility to define a radial force control can be useful when bearing damages happen or to prevent them. Bearing fault is one of the most frequent

G. Sala and A. Tani are with the Department of Electric, Electronic and Information Engineering, Bologna University, Bologna, Italy (e-mail: giacomo.sala5@unibo.it; angelo.tani@unibo.it).

C. Gerada and D. Gerada are with the Power Electronics, Machines and Control Group, Department of Electrical and Electronic Engineering, The University of Nottingham, Nottingham, NG7 2RD, U.K. (e-mail: chris.gerada@nottingham.ac.uk; david.gerada@nottingham.ac.uk). mechanical faults in electrical machines. To avoid dangerous consequences, it is possible to control the radial force to release the bearing from the rotor weight and compensate for other possible radial force sources. In a closed loop control, this makes it also possible to have a bearingless machine control.

The analysed machine drawing and winding are shown in Fig.1. The machine is a triple three-phase sectored SPM machine. The sectorization concept refers to a design in which the three-phase windings are supplied by different VSIs, and each of this resulting systems is located in a different stator area (sector), as in [5]-[7].

In [5] the radial force control for this machine has already been verified by a methodology based on the Single Value Decomposition (SVD) technique. In this paper, the radial force control is derived by the control of the harmonic fields in the airgap, based on the multi space vectors approach, as in [8]-[9].

This paper is the first part of a work on the radial force control for triple three-phase sectored SPM machines. In this first part (Part I) are presented: the radial force (Section II); the model for a triple three-phase machine, taking into account both the even and odd harmonic fields in the air-gap (Section III); the relation between the radial force and the current space vectors (Section IV); the FE simulations, used to verify the control technique (Section V). In the second part (Part II [10]) a fault tolerant control in case of one threephase sector open winding fault is analysed.

\section{RADIAL FORCE MODELING}

The first aim of this paper is to define a machine model to evaluate the radial force once the phase currents are known.

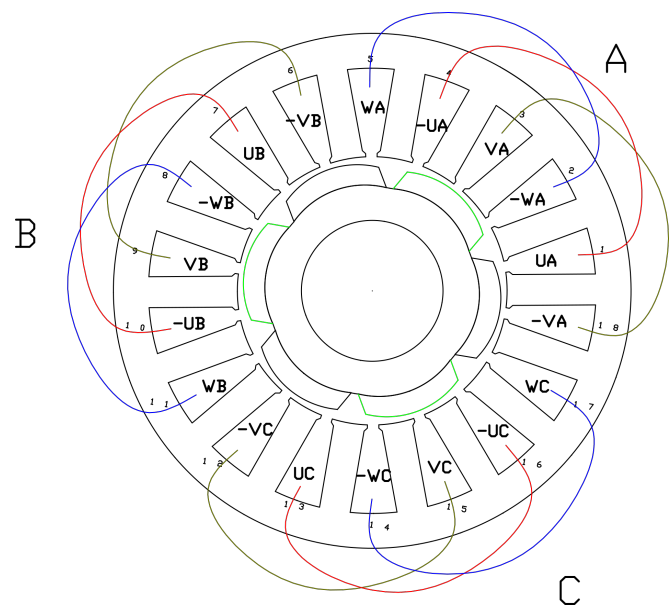

Fig. 1. Sectored triple three-phase machine stator winding configuration and drawing. 
The model is defined with the approximation of isotropic stator and radial flux in the airgap, neglecting the tangential component, as in [1] (if this component is not negligible, different approaches must be used, as in [4]-[5]). It is written in mechanical angles, and the time dependence is implicit. The rotor skew, the permanent magnet shape and the slot effect are not taken into account in the analytical model.

These approximations allow simplifying the machine equations and underline the qualitative advantages of the proposed triple three-phase machine control, neglecting an accurate quantitative evaluation of the machine parameters.

Under these assumptions, the resultant radial force in the machine can be described by the vector

$$
\bar{F}\left(\theta_{m}\right)=\int_{0}^{2 \pi} p_{r}\left(\theta_{s}, \theta_{m}\right) e^{j \theta_{s}} L R d \theta_{s},
$$

where $\theta_{m}$ is the angular position of the rotor, $\theta_{s}$ the angular position defined in the stator reference frame, $L$ the machine active length, $R$ the average air-gap radius and $p_{r}\left(\theta_{s}, \theta_{m}\right)$ is the pressure acting on the rotor in the infinitesimal arch centered in $\theta_{S}$. This pressure can be evaluated as

$$
p_{r}\left(\theta_{s}, \theta_{m}\right)=0.5 \mu_{0} H^{2}\left(\theta_{s}, \theta_{m}\right),
$$

where $H\left(\theta_{s}, \theta_{m}\right)$ is the air-gap field in the angular position $\theta_{s}$. The magnetic field is the sum of the armature contribution $H_{c u}$ and the permanent magnet one $H_{P M}$.

In linear condition the air-gap field can be defined as

$$
H\left(\theta_{s}, \theta_{m}\right)=H_{c u}\left(\theta_{s}\right)+H_{P M}\left(\theta_{s}, \theta_{m}\right) \text {. }
$$

These two components of the magnetic field distribution can be analyzed by their Fourier series

$$
\begin{aligned}
& H_{c u}\left(\theta_{s}\right)=\sum_{\rho=1}^{\infty} \Re\left\{\bar{H}_{c u, \rho}(t) e^{-j \rho \theta_{s}}\right\}, \\
& H_{P M}\left(\theta_{s}, \theta_{m}\right)=\sum_{\rho=1}^{\infty} \Re\left\{\bar{H}_{P M, s, \rho}\left(\theta_{m}\right) e^{-j \rho \theta_{s}}\right\},
\end{aligned}
$$

where $\rho$ is the harmonic index of the series. Substituting (2) in (1), and taking (3)-(5) into account, (1) can be rewritten as

$$
\bar{F}\left(\theta_{m}\right)=\bar{F}_{c u c u}+\bar{F}_{c u P M}\left(\theta_{m}\right),
$$

where $\bar{F}_{\text {cucu }}$ represents the radial force related to the interaction between the harmonic fields of the stator currents, and $\bar{F}_{c u P M}$ is the radial force due to the interaction between the permanent magnets and the armature field, defined as

$$
\begin{aligned}
& \bar{F}_{c u c u}=(\pi / 4) \mu_{0} L R \sum_{\rho=1}^{\infty}\left[\bar{H}_{c u, \rho}^{*} \bar{H}_{c u, \rho+1}+\bar{H}_{c u, \rho} \bar{H}_{c u, \rho-1}^{*}\right], \\
& \bar{F}_{c u P M}\left(\theta_{m}\right)=(\pi / 2) \mu_{0} L R \sum_{\rho=1}^{\infty}\left[\bar{H}_{P M, \rho} \bar{H}_{c u, \rho+1}^{*} e^{-j \rho \theta_{m}}+\bar{H}_{P M, \rho} \bar{H}_{c u, \rho-1}^{*} e^{j \rho \theta_{m}}\right]
\end{aligned}
$$

where $\mu_{0}$ is the permeability of free space, "**" is the complex conjugate and $\bar{H}_{P M, \rho}=\bar{H}_{P M, s, \rho} e^{-j \rho \theta_{m}}$ is the $\rho$-th harmonic representative vector of the permanent magnet field in the rotor reference frame. Note that, by substituting (2) and (3) in (1), it is possible to verify that the radial force contribution related to $H_{P M}{ }^{2}$ is the only one always null. This allows the model to take into account only the force components related to $2 H_{c u} H_{P M}$ and $H_{c u}{ }^{2}$.

\section{Multi Space Vector Model of a Triple Three-Phase SECTORED SPM MACHINE}

To analyze and control the radial force described by (6)(8), the magnetic field in the air-gap is analyzed. Because the armature field harmonics in (7) and (8) can be both even and odd, the machine model is developed to describe all the airgap field harmonics, as a function of the rotor angular position and the currents.

A multi-phase Clarke transformation is introduced, based on the idea that a nine-phase machine can be described like an eighteen one with a null current in half of the phases. The multi space vector transformation is defined as

$$
\bar{i}_{\rho}=\frac{2}{9} \sum_{x=1}^{N_{s}} i_{x} e^{j \rho \psi_{x}}, \quad[\rho=0,1,2, . ., \infty],
$$

where $N_{s}=18$ is the slot number, 9 is the phase number, $\psi_{x}=2 \pi / N_{s}(x-1)$ is the position of the magnetic axis of the winding starting from the $x$-th slot and ending in the $(x+3)$-th slot. It means that, following the winding scheme shown in Fig. 1, $i_{x}$ is the current flowing in the phase wound through the $x$-th slot when $x=18,1,2,6,7,8,12,13,14$, and $i_{x}=0$ for all the other $x$ values. In this way, the phase currents are related to the slot currents used in (9) by

$$
\begin{aligned}
& i_{18}=-i_{V A}, i_{1}=i_{U A}, i_{2}=-i_{W A}, \\
& i_{6}=-i_{V B}, i_{7}=i_{U B}, i_{8}=-i_{W B}, \\
& i_{12}=-i_{V C}, i_{13}=i_{U C}, i_{14}=-i_{W C} .
\end{aligned}
$$

The phase currents can also be represented by the standard three-phase Clarke transformation, since each three-phase winding of a sector is supplied by its own VSI. The threephase transformation, in a general X-th three-phase winding (with $\mathrm{X}$ equal to $\mathrm{A}, \mathrm{B}$ or $\mathrm{C}$ ), is

$$
\begin{aligned}
& \bar{i}_{X}=2 / 3\left(i_{U X}+i_{V X} \bar{\alpha}_{3 P}+i_{W X} \bar{\alpha}_{3 P}{ }^{2}\right), \\
& i_{X 0}=2 / 3\left(i_{U X}+i_{V X}+i_{W X}\right),
\end{aligned}
$$

and the related standard inverse - transformation is

$$
\begin{aligned}
& i_{U X}=i_{X 0} / 2+\mathfrak{R}\left\{\bar{i}_{X} \bar{\alpha}_{3 P}\right\}, \\
& i_{V X}=i_{X 0} / 2+\mathfrak{R}\left\{\bar{i}_{X} \bar{\alpha}_{3 P}^{2}\right\}, \\
& i_{W X}=i_{X 0} / 2+\Re\left\{\bar{i}_{X} \bar{\alpha}_{3 P}\right\},
\end{aligned}
$$

where $\bar{\alpha}_{3 P}=\exp (j 2 \pi / 3)$. Assuming that each three-phase subsystem has its own star connection ( $i_{X 0}$ is zero), it is also possible to define the triple three-phase $\rho$-th current space vector, defined by (9), as

$$
\bar{i}_{\rho}=1 / 3\left(\bar{i}_{m \rho} c_{m \rho}+\bar{i}_{n \rho}^{*} c_{n \rho}\right), \quad[\rho=0,1,2, . ., \infty],
$$

where

$$
\begin{aligned}
& \bar{i}_{m \rho}=1 / 3\left(\bar{i}_{A}+\bar{i}_{B} \bar{\alpha}^{6 \rho}+\bar{i}_{C} \bar{\alpha}^{12 \rho}\right), \\
& \bar{i}_{n \rho}=1 / 3\left(\bar{i}_{A}+\bar{i}_{B} \bar{\alpha}^{-6 \rho}+\bar{i}_{C} \bar{\alpha}^{-12 \rho}\right), \\
& c_{m \rho}=1-\bar{\alpha}^{(-6-\rho)}-\bar{\alpha}^{(-12+\rho)}, \\
& c_{n \rho}=1-\bar{\alpha}^{(6-\rho)}-\bar{\alpha}^{(12+\rho)},
\end{aligned}
$$

with $\bar{\alpha}=\exp \left(j 2 \pi / N_{s}\right)$. For the transformations (9) and (14), it is also possible to verify the relation

$$
\bar{i}_{\rho}=\bar{i}_{N_{s}+\rho}=\bar{i}_{N_{s}-\rho}^{*},[\rho=0,1,2, ., 9] .
$$

This relation allows defining the generic inversetransformation 


$$
i_{x}=\frac{i_{0}}{4}+\frac{i_{9}}{4}(-1)^{(x-1)}+\frac{1}{2} \sum_{\rho=1}^{8} \mathfrak{R}\left\{i_{\rho} e^{-j \rho \psi_{x}}\right\} .
$$

Two other inverse transformations, that can be used in order to define the machine control, are

$$
\begin{aligned}
& i_{x}=i_{0} / 2+\sum_{\rho_{\text {even }}=2}^{8} \mathfrak{R}\left\{i_{\rho} e^{-j \rho \psi_{x}}\right\}, \\
& i_{x}=i_{9}(-1)^{(x-1)} / 2+\sum_{\rho_{\text {odd }}=1}^{7} \mathfrak{R}\left\{i_{\rho} e^{-j \rho \psi_{x}}\right\} .
\end{aligned}
$$

Equation (22) is a suitable inverse transformation if only the odd spaces control is analyzed, as it is (21) for the even spaces control, because the currents are directly derived by a number of space vectors equal to the 9 degrees of freedom of nine-phase machines. To define a suitable technique for both the even and odd space vectors control, (20) should be used.

Because (20) needs a redundant number of space vectors, a new set of variables must be introduced to relate the space vectors in (21) with the space vectors in (22). Owing to the star connections, (14) can be analyzed, in each space, choosing from (15)-(16) the three current vectors

$$
\begin{aligned}
& \bar{i}_{M}=\bar{i}_{m 1}=1 / 3\left(\bar{i}_{A}+\bar{i}_{B} \bar{\alpha}_{3 P}+\bar{i}_{C} \bar{\alpha}_{3 P}{ }^{2}\right), \\
& \bar{i}_{N}=\bar{i}_{n 1}=1 / 3\left(\bar{i}_{A}+\bar{i}_{B} \bar{\alpha}_{3 P}{ }^{2}+\bar{i}_{C} \bar{\alpha}_{3 P}\right), \\
& \bar{i}_{S}=\bar{i}_{m 0}=\bar{i}_{n 0}=1 / 3\left(\bar{i}_{A}+\bar{i}_{B}+\bar{i}_{C}\right) .
\end{aligned}
$$

As shown in (23), the currents can be described by three complex variables (six scalar variables), and this result is coherent with the six degrees of freedom of a triple threephase machine. Therefore, the space vectors defined by (14)(18), and used in (21)-(22), can be written as

$$
\begin{aligned}
& i_{0}=2 / 3\left(\bar{i}_{S}+\bar{i}_{S}^{*}\right), \\
& \bar{i}_{1}=1 / 3\left(\bar{i}_{M} c_{m 1}+\bar{i}_{N}^{*} c_{n 1}\right), \\
& \bar{i}_{2}=1 / 3\left(\bar{i}_{N} c_{m 2}+\bar{i}_{M}^{*} c_{n 2}\right), \\
& \bar{i}_{3}=\bar{i}_{S}, \\
& \bar{i}_{4}=1 / 3\left(\bar{i}_{M} c_{m 2}+\bar{i}_{N}^{*} c_{n 4}\right), \\
& \bar{i}_{5}=1 / 3\left(\bar{i}_{N} c_{m 1}+\bar{i}_{M}^{*} c_{n 5}\right), \\
& \bar{i}_{6}=1 / 3\left(2 \bar{i}_{S}-\bar{i}_{S}^{*}\right), \\
& \bar{i}_{7}=1 / 3\left(\bar{i}_{N} c_{n 1}+\bar{i}_{M}^{*} c_{n 5}\right), \\
& \bar{i}_{8}=1 / 3\left(\bar{i}_{N} c_{n 2}+\bar{i}_{M}^{*} c_{n 4}\right), \\
& \bar{i}_{9}=0 .
\end{aligned}
$$

The introduction of a new set of transformation and space vectors is justified by the representation of (24)-(33), where it is possible to note that the new space vectors $\bar{i}_{M}, \bar{i}_{N}$ and $\bar{i}_{S}$ allow easily describing all the current space vectors related to the machine control.

By (14) the armature field harmonics can be easily defined as

$$
\bar{H}_{c u, \rho}=\frac{9 N_{c} \sin (\rho \pi / 6)}{\pi \delta \rho} \bar{i}_{\rho}, \quad[\rho=0,1,2, . ., \infty],
$$

while the permanent magnet field harmonics can be evaluated as

$$
\bar{H}_{P M, \rho}=12 \frac{B_{P M r} \delta_{P M}}{\pi \mu_{0} \delta \rho} \sin \left(\rho \frac{\Delta \Psi_{P M}}{2}\right),[\rho=3,9,15 . ., \infty],
$$

where $N_{c}$ is the turns number for each phase, $p$ the machine pole pairs number, $\delta$ and $\delta_{P M}$ the air-gap and magnet thicknesses, $B_{P M r}$ the residual flux density and $\Delta \Psi_{P M}$ the magnet angular amplitude (its maximum value is $\pi / 3$ ). Note that in (35) $\rho$ is a multiple of 3 and an odd number, otherwise the $\rho$-th permanent magnet harmonic is zero.

The generic $\rho$ - th space vector of the linked fluxes can be described as

$$
\bar{\phi}_{\rho}=\sum_{h=0}^{9} L_{\rho, h} \bar{i}_{h}+\bar{\phi}_{P M, \rho} \quad, \quad[\rho=0,1,2, . ., 9],
$$

where $L_{\rho, h}$ is the mutual inductance between the $h$-th space and the $\rho$-th space, $L_{\rho, \rho}$ the $\rho$-th space self-inductance, and $\bar{\phi}_{P M, \rho}$ is the $\rho$-th space vector of the permanent magnet linked fluxes. The machine phase voltage equations can be written in the multi space vector representation, as

$$
\bar{v}_{\rho}=r_{s} \bar{i}_{\rho}+l_{d s} \frac{d \bar{i}_{\rho}}{d t}+\frac{d \bar{\phi}_{\rho}}{d t}, \quad[\rho=0,1,2, \ldots, 9],
$$

where $r_{s}$ is the phase resistance, $l_{d s}$ the phase leakage inductance and $v_{\rho}$ the $\rho$-th space vector of the voltages. The transformations for the space vectors of the voltages and linked fluxes are defined in the same way as for the currents.

It can be verified that the instantaneous machine electrical power can be written as

$$
P=\sum_{x=1}^{N_{s}} v_{x} i_{x}=\frac{9}{2} \sum_{\rho o d d=1}^{7} \overline{v_{\rho}} \cdot \overline{i_{\rho}}
$$

with ". " the scalar product.

The electromechanical instantaneous power can be assessed by the analysis of the electrical power components. Moreover, introducing $i d_{3}+j i q_{3}=\overline{i_{3}} e^{-j p \theta_{m}}$, the main current space vector in the rotor reference frame, the machine torque can be evaluated by (38) as

$$
\begin{aligned}
& T_{m}=\left(\frac{9}{2}\right)^{2} L_{0 P M} \sum_{k=0}^{\infty}\left[\frac{j e^{j N_{S} k \theta_{m}}}{\left(N_{S} k+3\right)}+\frac{-j e^{-j N_{S}(k+1) \theta_{m}}}{\left(N_{S}(k+1)-3\right)}\right] \cdot j i q_{3} \\
& \text { with } L_{0 P M}=12 \frac{4}{9 \pi} N_{c} L R \frac{{ }_{P M r} \delta_{P M}}{\delta} \text {. }
\end{aligned}
$$

Equation (39) can be analyzed as sum of a main torque component (with $k$ equal to zero)

$$
T_{M}=\left(\frac{9}{2}\right)^{2} L_{0 P M}\left[\frac{j}{3}\right] \cdot j i q_{3},
$$

with the ripple term related to the positive sequence armature fields (the $7^{\text {th }}, 13^{\text {th }}, \ldots$ in electrical degrees)

$$
\sum_{k=1}^{\infty} T_{r\left(N_{S} k+3\right)}=\left(\frac{9}{2}\right)^{2} L_{0 P M} \sum_{k=1}^{\infty}\left[\frac{j e^{j N_{S} k \theta_{m}}}{\left(N_{S} k+3\right)}\right] \cdot j i q_{3},
$$

and the ripple terms related to the negative sequence armature fields (the $5^{\text {th }}, 11^{\text {th }}, \ldots$ in electrical degrees)

$$
\sum_{k=1}^{\infty} T_{r\left(N_{S} k-3\right)}=\left(\frac{9}{2}\right)^{2} L_{0 P M} \sum_{k=1}^{\infty}\left[\frac{-j e^{-j N_{S} k \theta_{m}}}{\left(N_{S} k-3\right)}\right] \cdot j i q_{3},
$$


The torque ripple amplitudes (related to $1 \mathrm{~A} i q_{3}$ current supply), " $T_{r}$ ", reported also in per unit to the main torque contribution) " $T_{M}$ ", and their frequencies (in times of the machine electrical frequency) are compared in Table I for the analyzed machine. It is possible to notice that the amplitudes of the positive and negative sequence torque ripple components are just a percentage of the main contribution. This allows simplifying the torque equation, for the machine speed control and behaviour, as

$$
T_{M}=\frac{27}{4} L_{0 P M} i q_{3}
$$

The analysis of the torque components has been reported, because the same approach is carried out in Section IV to investigate the radial force contributions. Furthermore, (39) shows that the torque can be controlled by only one degree of freedom, $i q_{3}$. It follows that in this machine it is not possible to improve the torque performance by a multiphase advanced control technique.

\section{RADIAL FORCE CONTROL}

Once the machine electromagnetic model is defined, it is possible to relate the current space vectors to the radial force contributions in order to well define the radial force control technique. As explained in Section III, it is possible to control three current space vectors. The main current space vector $\bar{i}_{3}$ (equal to $\bar{i}_{S}$ ) is needed to control the main torque component, and there are still four degrees of freedom (it means two space vectors) that can be used to control the radial force.

Introducing (34) and (35) in (7) and (8) allows evaluating (6). From the explication of the current space vectors in (6), the radial force contributions can be identified in

$$
\begin{aligned}
\bar{F}\left(\theta_{m}\right) & =\sum_{\rho=1}^{\infty}\left[K_{c u}(\rho, \rho+1)^{-{ }^{*}} \rho^{\bar{i}}(\rho+1)+K_{c u}(\rho, \rho-1)^{\bar{i}} \rho^{-*} \rho-1+\right. \\
& \left.+K_{P M}(\rho, \rho+1) \bar{i}(\rho+1) e^{-i \rho \theta_{m}}+K_{P M}(\rho, \rho-1)^{i}(\rho-1) e^{i \rho \theta_{m}}\right] .
\end{aligned}
$$

with

$$
\begin{aligned}
& K_{c u} \rho, h=\left(\frac{9 N_{c}}{\pi \delta}\right)^{2} \frac{\pi}{4} \mu_{0} L R \frac{\sin (\rho \pi / 6)}{\rho} \frac{\sin (h \pi / 6)}{h}, \\
& K_{P M} \rho, h=6 L R \frac{9 N_{c}}{\pi} \frac{B_{P M r} \delta_{P M}}{\delta^{2}} \frac{\sin (\rho \pi / 6)}{\rho} \frac{\sin (h \pi / 6)}{h} .
\end{aligned}
$$

The evaluation of the contribution of the different current space vectors (armature field harmonics) to the radial force is shown in Table II. As can be seen from Table II, the radial

\begin{tabular}{|c|c|c|c|c|c|c|c|}
\hline $\mathrm{K}$ & & \multicolumn{5}{|c|}{$T_{M}$ (iq=1A) [Nm] } & frequency pu \\
\hline 0 & & \multicolumn{5}{|c|}{0.55} & 0 \\
\hline & & \multicolumn{2}{|c|}{${ }^{T} r(18 k-3)$} & & \multicolumn{2}{|c|}{$T_{r(18 k+3)}$} & \\
\hline & & $\operatorname{Tr}(\mathrm{iq}=1 \mathrm{~A})[\mathrm{Nm}]$ & $\mathrm{pu}\left(\operatorname{Tr} / T_{M}\right)$ & & $\operatorname{Tr}(\mathrm{iq}=1 \mathrm{~A})[\mathrm{Nm}]$ & $\mathrm{pu}\left(\mathrm{Tr} / T_{M}\right)$ & \\
\hline 1 & $T_{r 3(5)}$ & 0.02 & 0.03 & $T_{r 3(7)}$ & 0.02 & 0.04 & 6 \\
\hline 2 & $T_{r 3(11)}$ & 0.01 & 0.02 & $T_{r 3(13)}$ & 0.01 & 0.02 & 12 \\
\hline 3 & $T_{r 3(17)}$ & 0.01 & 0.01 & $T_{r 3(19)}$ & 0.01 & 0.01 & 18 \\
\hline 4 & $T_{r 3(23)}$ & 0.00 & 0.01 & $T_{r 3(25)}$ & 0.01 & 0.01 & 24 \\
\hline
\end{tabular}
force is mainly produced by the interaction between the main permanent magnet harmonic field $\left(3^{\text {rd }}\right)$ and the $2^{\text {nd }}$ and $4^{\text {th }}$

TABLE I

TORQue CONSTANT VALUES AND RIPPLE FREQUENCIES. armature ones (with radial force coefficients of $23.8 \mathrm{~N} / \mathrm{A}$ and 11.9 N/A respectively). As done for the torque in (43), the force equation can be simplified as

$$
\bar{F}\left(\theta_{m}\right)=K_{P M 3,2} \bar{i}_{2}^{*} e^{j 3 \theta_{m}}+K_{P M 3,4} \bar{i}_{4} e^{-j 3 \theta_{m}} .
$$

Equations (43) and (47) allow decoupling the torque control by the radial force control.

If the reference force $\bar{F}\left(\theta_{m}\right)$ is equal to $F e^{i \theta_{f}}$, with $\theta_{f}$ the angular position of the reference radial force vector in the stator reference frame, the best control choice is the one with the two radial force components in (47) having the same direction, while their amplitudes can be modified to optimize the control or the minimization of Joule losses.

With this solution the resulting radial force vector is

$$
\bar{F}\left(\theta_{m}\right)=F e^{j \theta_{f}}=\left(F_{2}+F_{4}\right) e^{j \theta_{f}},
$$

where F2 and F4 represent how much the $2^{\text {nd }}$ and $4^{\text {th }}$ space vector contribute to the radial force. The $2^{\text {nd }}$ and $4^{\text {th }}$ current space vectors can be so evaluated from (47) and (48) as

$$
\bar{i}_{2}=\frac{F_{2}}{K_{P M 3,2}} e^{j\left(3 \theta_{m}-\theta_{f}\right)} \text {, and } \bar{i}_{4}=\frac{F_{4}}{K_{P M 3,4}} e^{j\left(3 \theta_{m}+\theta_{f}\right)} .
$$

The ratio $F 4 / F 2$ has been investigated in this paper, without defining an optimization algorithm. Once this ratio is chosen, the control is completely defined. As discussed in Section III, all the machine current vectors are easily defined by $\bar{i}_{M}, \bar{i}_{N}$ and $\bar{i}_{S}$, as shown in (24)-(33).

This three current space vectors can be evaluated by (26), (27) and (28) as

$$
\begin{aligned}
& \bar{i}_{S}=\bar{i}_{3}, \\
& \bar{i}_{M}=3 \frac{\bar{i}_{4}-\left(c_{n 4} / c_{m 2}\right) \bar{i}_{2}^{*}}{c_{m 2}-\left(c_{n 2} c_{n 4} / c_{m 2}\right)}, \\
& \bar{i}_{N}=3 \frac{\overline{i_{2}}-\left(c_{n 2} / c_{m 2}\right) \bar{i}_{4}^{*}}{c_{m 2}-\left(c_{n 2} c_{n 4} / c_{m 2}\right)} .
\end{aligned}
$$

All the machine currents can be calculated with these three current space vectors by (24)-(33) and (20)-(22). It is worth highlighting that all the spaces related to the torque generation (or to $\bar{i}_{S}$ ) are independent of the force ones (or of $\bar{i}_{M}$ and $\bar{i}_{N}$ ). The same result has been obtained for the space vectors of the linked fluxes. This result allows completely

TABLE II

FORCE CONSTANT VALUES.

\begin{tabular}{|c|c|c|c|c|c|c|c|c|c|}
\hline \multicolumn{5}{|c|}{ Currents Forces [N/A] } & \multicolumn{5}{c|}{ PMs with Currents Forces [N/A] } \\
\hline$\rho$ & \multicolumn{2}{|c|}{$K_{c u} \rho, \rho-1$} & \multicolumn{2}{|c|}{$K_{c u} \rho, \rho+1$} & $\rho$ & \multicolumn{2}{|c|}{$K_{P M}, \rho-1$} & \multicolumn{2}{|c|}{$K_{P M} \rho, \rho+1$} \\
\hline 1 & & & $K_{c u} 1,2$ & 0.075 & 3 & $K_{P M} 3,2$ & 23.800 & $K_{P M} 3,4$ & 11.900 \\
\hline 2 & $K_{c u} 2,1$ & 0.075 & $K_{c u} 2,3$ & 0.050 & 9 & $K_{P M} 9,8$ & 1.983 & $K_{P M} 9,10$ & 1.587 \\
\hline 3 & $K_{c u} 3,2$ & 0.050 & $K_{c u} 3,4$ & 0.025 & 15 & $K_{P M} 15,14$ & 0.680 & $K_{P M} 15,16$ & 0.595 \\
\hline 4 & $K_{c u} 4,3$ & 0.025 & $K_{c u} 4,5$ & 0.007 & 21 & $K_{P M} 21,20$ & 0.340 & $K_{P M 21,22}$ & 0.309 \\
\hline 5 & $K_{c u} 5,4$ & 0.007 & $K_{c u} 5,6$ & 0.000 & 27 & $K_{P M} 27,26$ & 0.203 & $K_{P M} 27,28$ & 0.189 \\
\hline 6 & $K_{c u} 6,5$ & 0.000 & $K_{c u} 6,7$ & 0.000 & 33 & $K_{P M} 33,32$ & 0.135 & $K_{P M} 33,34$ & 0.127 \\
\hline 7 & $K_{c u} 7,6$ & 0.000 & $K_{c u} 7,8$ & 0.003 & 39 & $K_{P M} 39,38$ & 0.096 & $K_{P M} 39,40$ & 0.092 \\
\hline 8 & $K_{c u} 8,7$ & 0.003 & $K_{c u} 8,9$ & 0.004 & 45 & $K_{P M} 45,44$ & 0.072 & $K_{P M} 45,46$ & 0.069 \\
\hline 9 & $K_{c u} 9,8$ & 0.004 & $K_{c u} 9,10$ & 0.003 & 51 & $K_{P M} 51,50$ & 0.056 & $K_{P M 51,52}$ & 0.054 \\
\hline 10 & $K_{c u} 10,9$ & 0.003 & $K_{c u} 10,11$ & 0.001 & 57 & $K_{P M} 57,56$ & 0.045 & $K_{P M} 57,58$ & 0.043 \\
\hline 11 & $K_{c u} 11,10$ & 0.001 & $K_{c u} 11,12$ & 0.000 & 63 & $K_{P M} 63,62$ & 0.037 & $K_{P M} 63,64$ & 0.035 \\
\hline 12 & $K_{c u} 12,11$ & 0.000 & $K_{c u} 12,13$ & 0.000 & 69 & $K_{P M} 69,68$ & 0.030 & $K_{P M} 69,70$ & 0.030 \\
\hline 13 & $K_{c u} 13,12$ & 0.000 & $K_{c u} 13,14$ & 0.001 & 75 & $K_{P M M} 75,74$ & 0.026 & $K_{P M} 75,76$ & 0.025 \\
\hline 14 & $K_{c u} 14,13$ & 0.001 & $K_{c u} 14,15$ & 0.001 & 81 & $K_{P M M} 81,80$ & 0.022 & $K_{P M} 81,82$ & 0.021 \\
\hline 15 & $K_{c u} 1,2$ & 0.001 & $K_{c u} 15,16$ & 0.001 & 87 & $K_{P M} 87,86$ & 0.019 & $K_{P M} 87,88$ & 0.019 \\
\hline
\end{tabular}


decoupling the control of the spaces related to the torque from those related to the force. Indeed, since the space vectors of the back-emfs are evaluated by (36), the voltage space vectors $\bar{v}_{\rho}$ in (37) can be defined as function of the rotor position $\theta_{m}$ and the current space vectors $\bar{i}_{\rho}$ (or $\bar{i}_{M}$, $\bar{i}_{N}$ and $\left.\bar{i}_{S}\right)$.

Finally, the current regulator on the $2^{\text {nd }}, 3^{\text {rd }}$ and $4^{\text {th }}$ spaces have to be synchronous with the $d$-axis of the rotor reference frame every time the reference force has a fixed direction: the $3^{\text {rd }}$, related to the torque control, by $\overline{i_{3}}=j i q_{3} e^{+j 3 \theta_{m}}$; the $2^{\text {nd }}$ and the $4^{\text {th }}$ related to the force by (49). Instead, if the force is dynamically controlled, for example to compensate for a dynamic eccentricity, and $d \theta_{f} / d t$ is equal to $\omega_{f}$, the $2^{\text {nd }}$ and $4^{\text {th }}$ space regulator reference frames rotate at $3 \omega_{m}-\omega_{f}$ and $3 \omega_{m}+\omega_{f}$ respectively.

\section{Simulation Results}

The analytical model of the triple three-phase machine and the radial force control technique are evaluated by $\mathrm{FE}$ simulations in Magnet ${ }^{\mathrm{TM}}$. The main data of the simulated machine are reported in Table III.

To match the model with the simulation results the main control parameters in (43) and (47) ( $K_{T R}, K_{P M 3,2}$ and $\left.K_{P M 3,4}\right)$ have been deduced by $\mathrm{FE}$ simulations. The knowledge of the radial force parameters is useful to control the radial force in open loop. Indeed, only with radial force sensors (or position ones) it is possible to define a closed loop control, and only in this case a high accuracy of the radial force parameters can be neglected. Instead, to optimize the ratio of the radial force components F2/F4, the radial force parameters must be known with enough accuracy even for a closed loop control.

The proposed FE simulation is conducted to validate the radial force control with different F2/F4 ratios. This has been carried out with a $5 \mathrm{Nm}$ reference torque and a speed of 3000 rpm. The machine open loop radial force control, with different F2/F4 ratios, is well presented in Figs. 2, 3 and 4.

The FE simulation in Fig. 2 describes a control with a

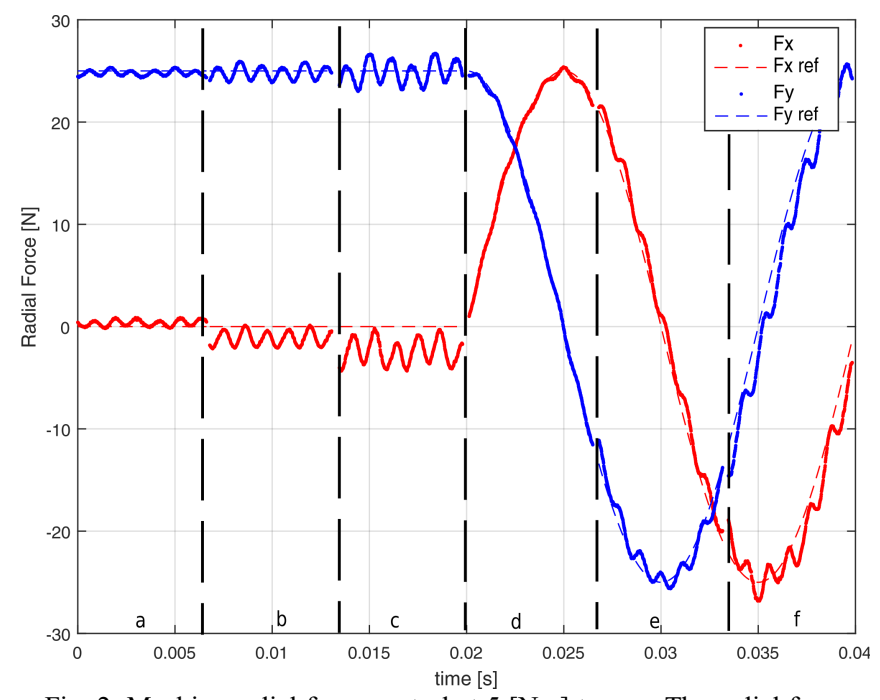

Fig. 2. Machine radial force control at $5[\mathrm{Nm}]$ torque. The radial force control is $25[\mathrm{~N}]$ static $(\mathrm{a}, \mathrm{b}, \mathrm{c})$ and $25[\mathrm{~N}]$ dynamic $(\mathrm{d}, \mathrm{e}, \mathrm{f})$. The ratio $\mathrm{F} 2 / \mathrm{F} 4$ is $0(\mathrm{a}, \mathrm{d}), 1(\mathrm{~b}, \mathrm{e})$ and infinite $(\mathrm{c}, \mathrm{f})$. constant force and then a force synchronous with the rotor. This can be used, for example, to compensate for a static or a dynamic rotor eccentricity.

This simulation is defined in an open loop control, and better results can be obtained in a closed loop control. It is also possible to note that, in order to reach a higher accuracy in the open loop force control, it is better to set a zero F2/F4 ratio (as in period a and $d$ in Fig. 2). This means that for higher accuracy it should be better to use only the $4^{\text {th }}$ current space vector in the radial force generation, and (47) becomes $\bar{F}=K_{P M 3,4} \bar{i}_{4} e^{-j 3 \theta_{m}}$. The control with the $2^{\text {nd }}$ current space vector (as in period $\mathrm{c}$ and $\mathrm{f}$ ) shows also a phase error, which is not present when the force is controlled by the $4^{\text {th }}$ one, but this error can be compensated as a phase offset. The $25 \mathrm{~N}$ radial force value has been chosen as a representative value to release the bearing from the rotor weight (the rotor mass is about $2.5 \mathrm{~kg}$ ). The torque resulting from the same simulation of Fig. 2 is shown in Fig. 3, where the reference torque is 5 $\mathrm{Nm}$, which is almost the rated torque of the machine. As shown in Fig. 3, the torque control can be independent of the radial force control, and the torque main value is always equal to the reference one when the torque constant is matched. The currents resulting from the same FE simulation are presented in Fig. 4, with regions 'a'-' $\mathrm{f}$ ' corresponding to the same time periods of Fig. 2 and Fig. 3.

In the shown radial force control the currents change is acceptable and can be maintained with withstand-able temperature arise. Without force control all the currents have a sinusoidal waveform with an amplitude of $11.6 \mathrm{~A}$. Instead, with the radial force control shown in Fig. 2 the peak current is $14.2 \mathrm{~A}$. The maximum current value depends on the radial force current space vectors ratio (F2/F4).

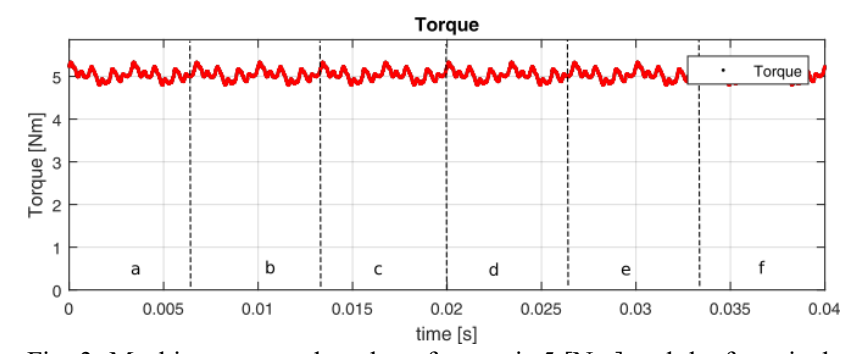

Fig. 3. Machine torque when the reference is $5[\mathrm{Nm}]$ and the force is the one in Fig. 2. The radial force control is $25[\mathrm{~N}]$ static $(\mathrm{a}, \mathrm{b}, \mathrm{c})$ and $25[\mathrm{~N}]$ dynamic $(\mathrm{d}, \mathrm{e}, \mathrm{f})$. The ratio $\mathrm{F} 2 / \mathrm{F} 4$ is $0(\mathrm{a}, \mathrm{d}), 1(\mathrm{~b}, \mathrm{e})$ and infinite $(\mathrm{c}, \mathrm{f})$.
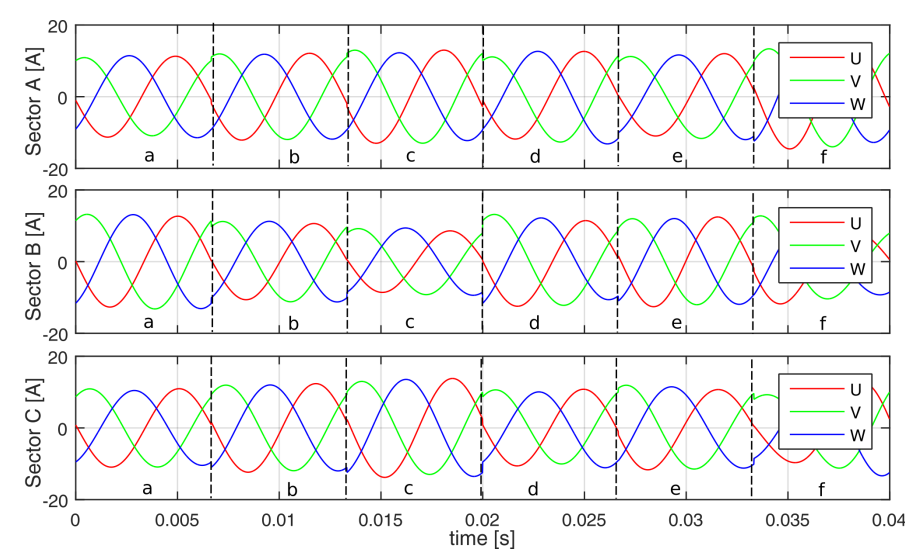

Fig. 4. Machine phase currents of Fig. 2 and Fig. 3 FE simulation. The radial force control is $25[\mathrm{~N}]$ static $(a, b, c)$ and $25[\mathrm{~N}]$ dynamic $(\mathrm{d}, \mathrm{e}, \mathrm{f})$. 
The F2/F4 ratio and the $d$-axis component of the main current space vector $i d_{3}$ in the rotor reference frame can be optimized to reduce the Joule losses or distribute them more equally among the phases, to minimize the peak current, to have higher control accuracy, or for other purposes.

\section{CONCLUSION}

A new model of a triple three-phase sectored SPM machine has been developed in order to define a radial force control decoupled from the torque one. The proposed technique can be used to release the bearings from a radial force and can be applied in bearingless applications, if suitable sensors are arranged. The radial force control and its independence of the torque control have been verified by FE analysis. The obtained results validated the possibility to neglect the tangential component of the magnetic field for a qualitative analysis of the radial force, and for the analyzed machine. Some solutions to improve the radial force control have been presented and others have been proposed.

\section{REFERENCES}

[1] S. Serri, A. Tani and G. Serra, "Analytical model of radial forces considering mutual effects between torque and levitation current space vectors in 5-phase PM bearingless motors," Industrial Electronics Society, IECON 2013 - 39th Annual Conference of the IEEE, Vienna, Austria, 2013, pp. 5142-5147.

[2] M. Ramezani and O. Ojo, "The modeling and position-sensorless estimation technique for a nine-phase interior permanent-magnet machine using high-frequency injections," in IEEE Transactions on Industry Applications, vol. 52, no. 2, pp. 1555-1565, March-April 2016.

[3] O. Ojo and M. Ramezani, "Sensorless speed control of symmetrical triple-star nine-phase Interior Permanent Magnet machines," 2016 IEEE Applied Power Electronics Conference and Exposition (APEC), Long Beach, California (USA), 2016, pp. 2035-2042.

[4] S. Serri, A. Tani and G. Serra, "A method for non-linear analysis and calculation of torque and radial forces in permanent magnet multiphase bearingless motors," Power Electronics, Electrical Drives, Automation and Motion (SPEEDAM), 2012 International Symposium on, Sorrento, Italy, 2012, pp. 75-82.

[5] G. Valente, L. Papini, A. Formentini, C. Gerada and P. Zanchetta, "Radial force control of multi-sector permanent magnet machines," 2016 XXII International Conference on Electrical Machines (ICEM), Lausanne, Switzerland 2016, pp. 2595-2601.

[6] M. Barcaro, N. Bianchi and F. Magnussen, "Faulty operations of a PM fractional-slot machine with a dual three-phase winding", IEEE Trans. Ind. Electron., vol. 58, no. 9, pp.3825 -3832, 2011.

[7] M. Barcaro, N. Bianchi and F. Magnussen, "Analysis and tests of a dual three-phase 12-slot 10-pole Permanent-Magnet motor," IEEE Transactions on Industry Applications, vol. 46, no. 6, pp. 2355-2362, Nov.-Dec. 2010.

[8] A. Tani, M. Mengoni, L. Zarri, G. Serra and D. Casadei, "Control of multiphase induction motors with an odd number of phases under open-circuit phase faults," in IEEE Transactions on Power Electronics, vol. 27, no. 2, pp. 565-577, Feb. 2012.

[9] A. Tani, Y. Gritli, M. Mengoni, L. Zarri, G. Sala, A. Bellini and G. Serra, "Detection of magnet demagnetization and high-resistance connections in five-phase surface-mounted permanent magnet generators," Diagnostics for Electrical Machines, Power Electronics and Drives (SDEMPED), 2015 IEEE 10th International Symposium on, Guarda, Portugal, 2015, pp. 487-493.

[10] G. Sala, D. Gerada, C. Gerada and A. Tani, "Radial force control for triple three-phase sectored SPM machines. Part II: open winding fault tolerant control," to be presented in: 2017 IEEE Workshop on Electrical Machines Design, Control and Diagnosis (WEMDCD), Nottingham, United Kingdom, 2017.
TABLE III

\begin{tabular}{ll} 
MAIN PARAMETERS OF THE ANALYZED NINE-PHASE SPM MACHINE [5]. \\
\cline { 1 - 2 } Pole number $(2 p)$ & 6 \\
PM material & NdFeB \\
kW Rating & $1.5 \mathrm{~kW}$ \\
Turn/coil & 22 \\
No Load Flux/sector & $0.026 \mathrm{~Wb}$ \\
Rated Speed & $3000 \mathrm{rpm}$ \\
Torque constant & $0.128 \mathrm{Nm} / \mathrm{A}$ \\
Voltage constant & $6.022 \mathrm{~V} / \mathrm{krpm}$ \\
Phase resistance $\left(\mathrm{R}_{\mathrm{ph}}\right)$ & $0.0808 \mathrm{Ohm}$ \\
d-axis Inductance/sector $(\mathrm{Ld})$ & $0.445 \mathrm{mH}(\mathrm{A}, 1 \mathrm{~A})$ \\
q-axis Inductance/sector $(\mathrm{Lq})$ & $0.442 \mathrm{mH}(@ 1 \mathrm{~A})$ \\
\hline Outer Stator diameter & $95 \mathrm{~mm}$ \\
Axial length & $91 \mathrm{~mm}$ \\
Air-gap length & $1 \mathrm{~mm}$ \\
Magnets thickness & $4 \mathrm{~mm}$
\end{tabular}

\section{BIOGRAPHIES}

Giacomo Sala received the M. Sc. degree in Electrical Engineering, with honors, in 2014 from the University of Bologna, Bologna, Italy. Since 2014 he has been working toward the Ph.D. degree at the Department of Electrical, Electronic and Information Engineering, University of Bologna. His research interests include fault tolerant and sensorless control of multiphase drives, and fault diagnosis of electrical machines.

David Gerada received the B.Eng.(Hons.) degree in electrical engineering from the University of Malta, Malta in 2007 and the Ph.D. degree in high-speed electrical machines from the University of Nottingham, Nottingham, UK in 2012. From 2007-2015 he was with the R\&D Department at Cummins Generator Technologies, Stamford, UK, first as an Electrical Machine Design Engineer (2007-2011) and then as a Senior Electrical Machine Design Engineer and Innovation Leader (2011-2016). In 2016 he joined the University of Nottingham as a Senior Research Fellow in Electrical Machines. His research interests include high speed machines, traction machines, use of novel materials, and multiphysics-based optimization of electrical machines. Dr. Gerada is a Chartered Engineer in the U.K. and a member of the Institution of Engineering and Technology.

Chris Gerada (SM'14) received the Ph.D. degree in numerical modeling of electrical machines from The University of Nottingham, Nottingham, U.K., in 2005. He was a Researcher with The University of Nottingham, working on high-performance electrical drives and design and modeling of electromagnetic actuators for aerospace applications. He became a Lecturer in Electrical Machines in 2008, an Associate Professor in 2011, and a Professor in Electrical Machines in 2013 at The University of Nottingham. He currently holds the Royal Academy of Engineering/Cummins Chair in Electrical Machines at The University of Nottingham. His research interests include the design and modeling of highperformance electric drives and machines. Prof. Gerada is an Associate Editor for the IEEE TRANSACTIONS ON INDUSTRY APPLICATIONS and is the Chair of the IEEE Industrial Electronics Society Electrical Machines Technical committee.

Angelo Tani was born in Faenza, Italy, in 1963. He received the M. Sc. in Electrical Engineering, with honors, from the University of Bologna, Bologna, Italy, in 1988. Currently he is an Associate Professor with the Department of Electric, Electronic and Information Engineering, University of Bologna. He has authored more than 150 journals and conference proceedings. His current activities include multiphase motor drives, ac/ac matrix converters, and field weakening strategies for induction motor drives. 